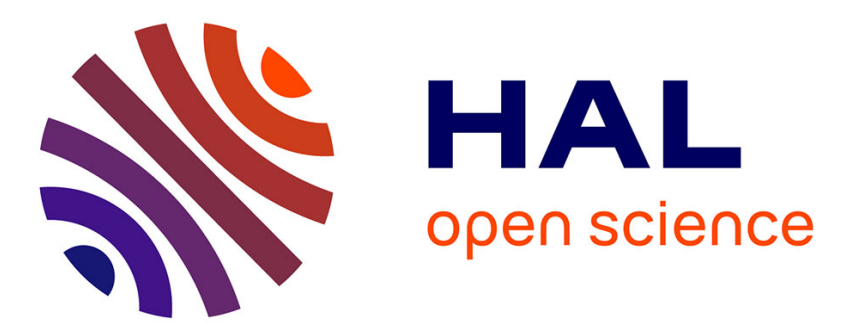

\title{
The road to crossovers: plants have their say
}

Christine Mézard, Julien Vignard, Jan Drouaud, Raphaël Mercier

\section{To cite this version:}

Christine Mézard, Julien Vignard, Jan Drouaud, Raphaël Mercier. The road to crossovers: plants have their say. Trends in Genetics, 2007, 23 (2), pp.91-99. 10.1016/j.tig.2006.12.007 . hal-02668854

\section{HAL Id: hal-02668854 https: / hal.inrae.fr/hal-02668854}

Submitted on 31 May 2020

HAL is a multi-disciplinary open access archive for the deposit and dissemination of scientific research documents, whether they are published or not. The documents may come from teaching and research institutions in France or abroad, or from public or private research centers.
L'archive ouverte pluridisciplinaire HAL, est destinée au dépôt et à la diffusion de documents scientifiques de niveau recherche, publiés ou non, émanant des établissements d'enseignement et de recherche français ou étrangers, des laboratoires publics ou privés. 
The road to crossovers: plants have their say

\section{Christine Mézard, Julien Vignard, Jan Drouaud and Raphaël Mercier}

Station de Génétique et d'Amélioration des Plantes, Institut Jean Pierre Bourgin, INRA, 78026 Versailles cedex, France

\begin{abstract}
Crossovers involve the reciprocal exchange of large fragments of genetic material between homologous chromosomes during meiosis. In this way, crossovers are the basis of genetics. Remarkably, the number and distribution of crossovers on chromosomes are closely controlled. Data from various model organisms (notably Saccharomyces cerevisiae) show that the distribution of crossovers results from a series of tightly regulated events involving the formation and repair of doublestrand breaks and interference. Recent advances in genetic and cytological tools, particularly for studying Arabidopsis thaliana, have enabled crossover control in plants to be studied in more detail. In this article, we discuss the contribution of plant studies to meiosis research, particularly to our understanding of crossover control and interference, and we evaluate models of interference.
\end{abstract}

\section{Introduction}

The crossover (see Glossary) and its cytological signature, the chiasma, are major features of genetics. Our knowledge of the molecular mechanisms of crossover formation has increased considerably in the past decade, owing to studies of fungi [1,2]. Nevertheless, little is known about controlling the number of crossovers and their distribution along chromosomes, except for the remarkable observation that crossovers and/or chiasma are not randomly distributed [3]. However, it has been observed that when this control is affected, the missegregation of chromosomes markedly increases, resulting in aneuploid gametes in organisms as diverse as yeast, nematodes, mammals and plants [4]. The current view is that the distribution of crossovers results from three constraints. First, each pair of chromosomes, regardless of size, has at least one chiasma (known as the obligatory chiasma), which is essential for the segregation of homologous chromosomes at the first meiotic division. Second, crossovers are not independent of each other: the occurrence of one crossover inhibits the occurrence of another such event in a distance-dependent manner, resulting in crossovers being spaced more evenly along the chromosomes than would be expected if they occurred independently. This phenomenon is known as 'positive' interference and is referred to as interference throughout this article [3]. Third, separately from the influence of interference, the densities of crossovers along chromo-

Corresponding authors: Mézard, C. (mezard@versailles.inra.fr) Mercier, R.(rmercier@versailles.inra.fr).

Available online 8 January 2007. somes vary greatly, so there is a nonhomogeneous distribution of crossovers.

Plants have always been at the forefront of the study of heredity (Box 1). Now, plant models can be used to study meiosis and recombination. This is because they not only enable use of the rare combination of both genetic and cytological approaches but also, owing mainly to technological progress with Arabidopsis thaliana, a full range of molecular approaches can be used. The data obtained in plants contribute to our general understanding of meiosis,

\section{Glossary}

Chiasma: the cytological signature of a crossover. Chiasmata are observed as connections between the homologous chromosomes in bivalents during meiosis, from diakinesis to metaphase I.

Crossovers: one of the products of meiotic DSB repair. The repair process, through breaking and rejoining DNA molecules, results in the reciprocal exchange of large fragments of genetic material (i.e. the exchange of homologous regions). Therefore, the genetic outcome of crossovers is the reassociation of genetic markers located on both sides of the crossover point. Also called crossing-overs.

Gene conversion: the nonreciprocal exchange of small fragments of genetic material (i.e. homologous regions), resulting in a non-mendelian segregation of genetic markers. Gene conversions are formed by the repair of a short region around a DSB site by homologous recombination, using the chromatid of the homologous partner chromosome as a template. They can be 'single' or associated with a crossover.

Leptotene: the first of the four substages of prophase in the first meiotic division (i.e. prophase I). During this substage, individual chromosomes condense into long strands. Initiation of recombination (i.e. DSBs) occurs at this stage.

Noncrossovers: the subset of meiotic DSB-repair events that is not associated with crossovers. Noncrossovers can be genetically detectable, in which case they are called single gene-conversion events. Whether noncrossovers are genetically detectable depends on the presence or absence of a polymorphism in the conversion tract (i.e. the fragment of DNA that has been exchanged in the process).

Pachytene: the third of the four substages of prophase in the first meiotic division (i.e. prophase I). This substage is characterized by completion of the polymerization of the synaptonemal complex along homologous chromosomes. Recombination is completed at this stage.

Recombination nodules: electron-dense structures located on chromosomes. These are observed in electron micrographs of meiotic chromosomes during prophase I. During zygotene, the early nodules (ENs) have a variable shape: for example, round and small in tomato (Solanum lycopersicum), and ellipsoidal and spherical in Allium species. These nodules disappear early in pachytene. The nodules that remain at pachytene are called late nodules (LNs), and they are larger and are usually ellipsoidal.

Synaptonemal complexes: structures that are specific to meiosis. By electron microscopy, a synaptonemal complex appears as a ladder-like structure that forms at zygotene, and formation of this complex is completed at pachytene. The two uprights of the ladder consist of each axial element (the continuous protein structures formed along each chromosome at leptotene) of the two homologous chromosomes (at this stage called lateral elements), and the rungs consist of a dimer of the protein Zip1. The function of synaptonemal complexes has not been determined.

Zygotene: the second of the four substages of prophase in the first meiotic division (i.e. prophase I). This substage is characterized by formation of the synaptonemal complex, which progressively links closely the pairs of homologous chromosomes. Recombination progresses during this stage. 


\section{Box 1. Genetics in plants: from Pisum sativum to} Arabidopsis thaliana

Since Mendel established the law of segregation of independent characteristics using the pea (Pisum sativum), plants have been at the heart of the history of genetics. Mendel's work was ignored for three decades, until his laws were rediscovered independently through studies of a large range of plants, including Trifolium pratense (red clover), Silene latifolia subsp. alba (white campion), Papaver somniferum (opium poppy), Zea mays (maize) and $P$. sativum $[69,70]$. The first partial linkage was found in Lathyrus odoratus (sweet pea) [71]. Although the data were not initially interpreted as a meiotic reassociation of characteristics, this was the first report of a genetic crossover. The first demonstration that crossovers are associated with physical recombination of chromosomes was in Z. mays [72], and the first evidence for chiasmata interference, predicted from crossover interference, was obtained in Vicia faba (fava bean) $[70,73]$.

Plants have been fundamental in numerous other genetic discoveries. Mobile DNA elements were first reported in $Z$. mays [74]. RNA-mediated silencing was first discovered in transgenic Petunia hybrida, and plants still have a central role in deciphering the underlying mechanisms of gene silencing [75]. One of the current favorite models of plant geneticists is Arabidopsis thaliana because of its suitability for combined molecular genetic and cytological analyses.

particularly owing to the comparisons that can be made with data obtained in other model organisms. Here, we review recent findings on crossovers in plants, particularly those concerning key genes in Arabidopsis, and we relate these findings to the numerous genetic and cytological data from other species, including yeast, on the distribution of crossovers and the role of interference.

\section{Different manifestations of recombination: from yeast to plants}

Recombination was first defined as the generation of a new combination of genes [5]. Now, this definition can be extended to a reassortment of markers. In meiosis, this can arise either by crossover or by 'single' gene conversion. Recently, the term noncrossover has been introduced to designate DNA double-strand break (DSB)-repair events that are not associated with crossovers. Noncrossovers include single gene-conversion events and events that are not genetically detectable. Crossover and noncrossover events are products of the repair of programmed DSBs [6]. It is also probable that some repair events occur on the sister chromatid [sister chromatid exchange (i.e. SCE)]; however, these events are difficult to analyze, so their frequency is not easy to estimate [7]. In budding yeast (Saccharomyces cerevisiae), meiotic DSBs are caused by a set of 11 proteins, including Spo11, which directly generates the DSBs [6] (Figure 1). Only four of these proteins (Spo11, Mre11, Rad50 and Ski8) are conserved in Arabidopsis. However, there are three Spo11 homologs in Arabidopsis. The Arabidopsis proteins AtSPO11-1 and AtSPO11-2 are required to initiate meiotic recombination $[8,9]$. Mre11 and Rad50 are involved in both the formation and the repair of DSBs in yeast, but their orthologs are not required for DSB formation in Arabidopsis [10,11]. It is also clear that the homolog of Ski8 is not required for meiosis in plants [12]. Although the mechanism of initiation of DSB formation is conserved across all species, there are clear differences in the control of this process.
Numerous data on crossover distribution in plants are available, largely because crossovers are the basis of genetic maps. By contrast, information about noncrossover distribution is poor, because these events are difficult to visualize by classical genetic analyses and have no (or little) impact on genetic maps. Crossovers can also be studied by cytology, visualized as chiasmata or as late recombination nodules $(\mathrm{LNs})$. Chiasmata reflect the same molecular event as crossovers [3] and are powerful and easy markers for counting the number of crossovers per cell and per chromosome. However, counting in this way could result in a slight underestimation, because, even in plants with large genomes, chiasmata that are close together cannot be resolved unambiguously [13]. Individual chiasmata can be visualized only in a few organisms, such as grasshoppers [3]. LNs lie on the central region of synaptonemal complexes during pachytene and closely reflect the sites of crossovers, providing a much better resolution than that provided by chiasmata $[14,15]$. However, the technique for efficient visualization of LNs is applicable to only a few species. At an earlier substage in prophase I (zygotene), there is a much larger population of nodules, called early recombination nodules (ENs). Their distribution, their protein content and the timing of their appearance indicate that ENs might mark the sites of recombination intermediates [14]. The recombinases Rad51 and Dmc1 are involved in meiotic DSB repair and colocalize with ENs [16]. These two proteins are not observed in all ENs at a given time, but this is probably because ENs have progressed through the process of DSB repair to different extents. The genes ATRAD51 and ATDMC1 have been functionally characterized in Arabidopsis. As expected, the atrad51 mutant shows strong meiotic fragmentation of its chromosomes [17]. Surprisingly, in Arabidopsis atdmc1 mutants, DSBs seem to be repaired using the sister chromatid, although they are not repaired in similar yeast mutants $[18,19]$. Another interesting difference is that recombination defects result in apoptosis in mammals, whereas, in Arabidopsis, meiosis progresses regardless of the defect, allowing access to more information about the mutant phenotype.

\section{Crossover frequency varies along chromosomes}

In all eukaryotes that have been studied, including plants, the distribution of crossovers or LNs along chromosomes is not homogeneous $[15,20]$, on either a megabase or a kilobase scale (Figure 2). That is, the local probability of a crossover varies among different chromosome intervals. This nonhomogeneity is the basis of the definition of 'hot' and 'cold' regions (which have significantly high and low crossover frequencies, respectively). A general rule is that centromeres are cold regions [21].

Plants have diverse chromosome sizes and structures, and, in each type of plant, these hot and cold regions are distributed in a particular way. In some plants - including wheat (Triticum aestivum), maize (Zea mays) and barley (Hordeum vulgare) - the crossover frequency tends to increase with the relative physical distance from the centromere. By contrast, in Allium fistulosum (Welsh onion), crossovers seem to cluster close to centromeres [3]. For other plants - for example, Arabidopsis, rice (Oryza sativa) 


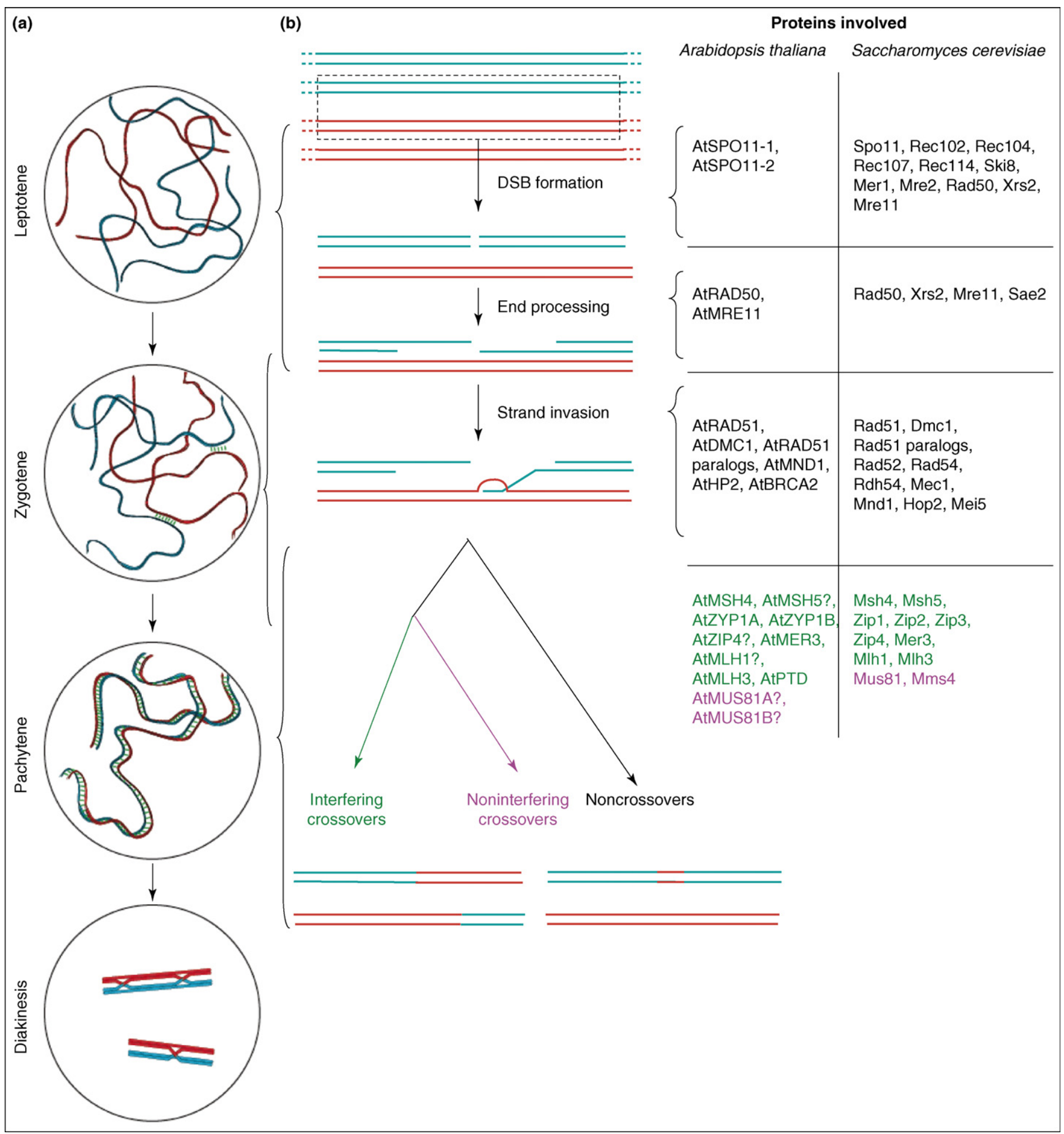

Figure 1. Prophase I of meiosis. (a) The cytological progression of prophase I of meiosis. After replication, chromosomes condense into long threads. This stage is known as leptotene. Then, at zygotene, the pairs of homologous chromosomes start to synapse, through formation of the synaptonemal complex. Next, at pachytene, synapsis is completed, and the synaptonemal complex links the homologs along their whole length. Then, at diakinesis (the final stage of prophase I), the synaptonemal complex has disappeared, and the further condensation of chromosomes reveals the presence of chiasmata. (b) A model of meiotic recombination pathways in Saccharomyces cerevisiae (yeast) and Arabidopsis thaliana. DNA DSBs are formed at leptotene and are processed to form single strands, which then invade a chromatid of the homologous chromosome as a repair template. Repair results in three different products: interfering crossovers, noninterfering crossovers and noncrossovers. Proteins involved in this process are indicated, together with the stage they are thought to operate at; data taken from Ref. [78]. Question marks indicate Arabidopsis proteins that are thought to be involved in this process on the basis of their similarity with S. cerevisiae proteins but that have not been functionally characterized. Alternative names for $S$. cerevisiae and Arabidopsis proteins are indicated here in parentheses: Rec107 (Mer2), Ski8 (Rec103), Mre2 (Nam8), Sae2 (Com1), Zip4 (Spo22), AtMSH5 (At3G20475), AtMUS81A (At4G30870) and AtMUS81B (At5G39770).

and tomato (Solanum lycopersicum) - crossover distribution varies between and along chromosome arms, with no apparent rule. Interestingly, within regions (cold or hot), crossover rates vary enormously from one kilobase to another in Arabidopsis [20] (Figure 2). These observations led to the hypothesis that there are several levels of control, each operating at a different scale: chromosomal, regional (megabase) and local (kilobase). 


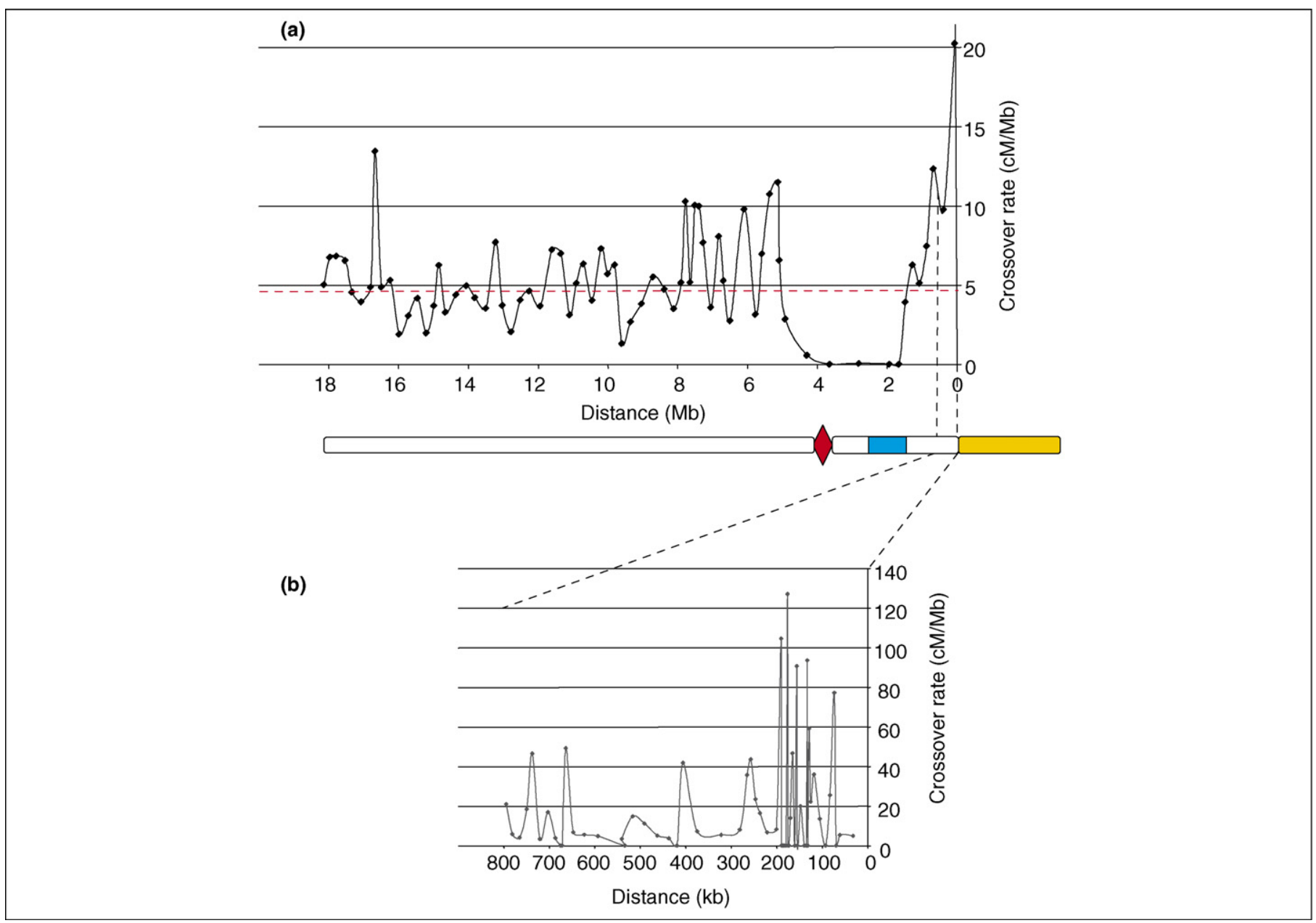

Figure 2. Variation in crossover rate along chromosome 4 of Arabidopsis thaliana. (a) The graph shows the variation in crossover rate along chromosome 4 of Arabidopsis A schematic representation of chromosome 4 is aligned with the graph, showing the centromere (red), the heterochromatic knob (blue) and the nucleolar organizer region (yellow). Part (a) reproduced, with permission, from Ref. [79] @ (2006) Cold Spring Harbor Laboratory Press. (b) The graph shows the variation in crossover rate in the first

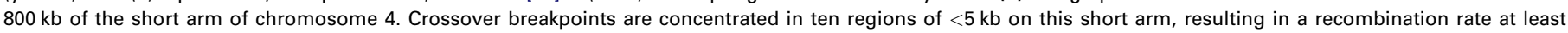
fivefold greater than the whole-chromosome average [4.6 centimorgan (cM)/Mb; dashed red line in (a)]. Part (b) reproduced, with permission, from Ref. [20] $($ (2006) the Biochemical Society.

In contrast to crossovers, the density of early recombination events, marked by ENs, is less variable along chromosomes [15,22]. ENs, or foci of either Rad51 or Dmc1, are often much more numerous than crossovers, strongly suggesting that DSBs are mostly repaired by noncrossovers. For example, there are 40 -fold more ENs than LNs in Allium species [14], and there are tenfold more Rad51 or Dmc1 foci than crossovers in the mouse [23]. In Arabidopsis, the ratio of AtRAD51 foci to crossovers is $\sim 25: 1$ [24], and models developed to infer genetic recombination rates from population genetic data fit better with a 1:1 ratio of gene conversion to crossovers [25]. The 1:1 estimate needs to be taken with caution, because it could be biased by several parameters (e.g. mutation and recombination rates, and sampling). In addition, this analysis detects only gene conversion and not 'silent' noncrossovers. The AtRAD51 foci probably provide a better estimate of the noncrossover to crossover ratio, even if they might mark other events (e.g. SCE).

The EN to LN ratio varies from interval to interval (from $4: 1$ to $20: 1$ in maize) $[14,26]$, indicating that all early recombination events have different probabilities of becoming a crossover. This point is also illustrated by the noncrossover to crossover ratio varying substantially from one locus to another in organisms as different as yeast and humans [27-29]. Therefore, the variation of crossover frequencies along a chromosome depends greatly on the choice of DSB-repair mechanism (crossover versus noncrossover), not only on the DSB distribution per se.

\section{Interference shapes the distribution of crossovers}

In addition to being unevenly distributed along chromosomes, crossovers are not independent of each other, because of the phenomenon of interference. Interference was defined by Muller in 1916 [30]: 'The occurrence of one crossing-over interferes with the coincident occurrence of another crossing-over in the same pair of chromosomes, and I have accordingly termed this phenomenon "interference".' An obvious consequence of interference is that crossovers are more distant from each other than would be expected if they were independent. Interference is commonly estimated genetically by the coefficient of coincidence [30] or cytologically by the distribution of LNs or chiasmata [31] (Box 2). The existence of interference has been confirmed in most species that have been tested; exceptions include Schizosaccharomyces pombe 
The first objective measurement of crossover interference, the coefficient of coincidence $(C)$, was described by Muller [30]. It is the ratio of the observed frequency of crossovers being simultaneously present in two different intervals to the product of the frequency of crossovers in each interval. Its value ranges from 1, when the occurrence of a crossover has no effect on the occurrence of another crossover (i.e. no interference), to 0 , when the presence of a crossover prohibits the occurrence of another crossover (i.e. total interference). Between these values, $C$ indicates the strength of interference.

There are several reports of negative interference $(C>1)$. We suggest that this type of interference falls into two categories. At short distances (a few kilobases), a noncrossover can be misinterpreted as two crossovers. When observed between two distant intervals, negative interference could be a side-effect of positive interference, resulting from an increased probability of having a second crossover at a distance corresponding to the end of the effect of positive interference $[76,77]$.

Plotting $C$ between each pair of intervals as a function of genetic distance yields a coincidence function that gives an overall view of interference [77] (as modeled in Figure lb).

Among several mathematical models that have been elaborated to describe this coincidence function, gamma models turned out to provide the best fit between experimental and predicted $C$ values [77]. Indeed, gamma models can be used to provide a distribution of the distances between crossovers (i.e. the inter-crossover distances; Figure la) from which a coincidence function can be deduced (Figure Ib). The probability density function of the gamma distribution in Figure la is shown in Equation I:

$$
f(x ; v, \lambda)=\frac{\lambda^{v} \boldsymbol{x}^{(v-1)} e^{-\lambda x}}{\Gamma(v)}
$$

where $x$ is the input variable, $v$ is a shape parameter, $\lambda$ is a rate parameter, and $\Gamma(v)$ is the gamma function; the mean is $v / \lambda$, and the variance is $v / \lambda^{2}$.

The shape of a gamma distribution curve is described by the parameter $v$ [58], which, biologically, reflects the number of noncrossovers between two crossovers plus one $(m+1)$. When $v$ is 1 , the distribution of the intercrossover distances is exponential (Figure la), and the coincidence function is a horizontal straight line that has a value of 1 (Figure lb). That is, for $v=1$, intercrossover distances are random, so there is no interference. As $v$ increases, the distribution of the intercrossover distances tends to be gaussian (Figure la), and the $C$ values in the deduced coincidence functions decrease at short distances (Figure $\mathrm{lb}$ ). In other words, for high values of $v$, the intercrossover distances are constrained and become less variable, and interference is strong.

(fission yeast) and Aspergillus nidulans (also known as Emericella nidulans) [15]. For example, in tomato and maize, the observed distribution of the distance between two LNs is different from that expected if their positions were assumed to be independently and homogeneously distributed [31,32]. In addition, detailed analyses of crossovers in yeast, humans and Arabidopsis strongly indicate that although most crossovers are subject to interference, some are not [33-37]. The existence of two classes of crossover (one subject to interference and one not) has been confirmed by mutant analyses in yeast $[1,2]$ and Arabidopsis [38-41].

\section{Formation of interfering crossovers}

In yeast, interfering-crossover formation is catalyzed by the recruitment of a set of meiosis-specific proteins referred to as ZMM proteins [for Zip1, Zip2, Zip3 (also known as Cst9), Msh4, Msh5 and Mer3 (also known as Hfm1) proteins] [42] (Figure 1) - to a subset of DSB sites. Noninterfering crossovers depend on the proteins Mus81
Therefore, $v$ is a measure of the strength of interference. It can be estimated either by finding the $v$ value that provides the best fit for the observed distribution of intercrossover distances (measured genetically or cytologically [58]) or from observed $C$ values [64]. The gamma model can be used as a tool to measure interference without assuming any particular biological mechanism [58].
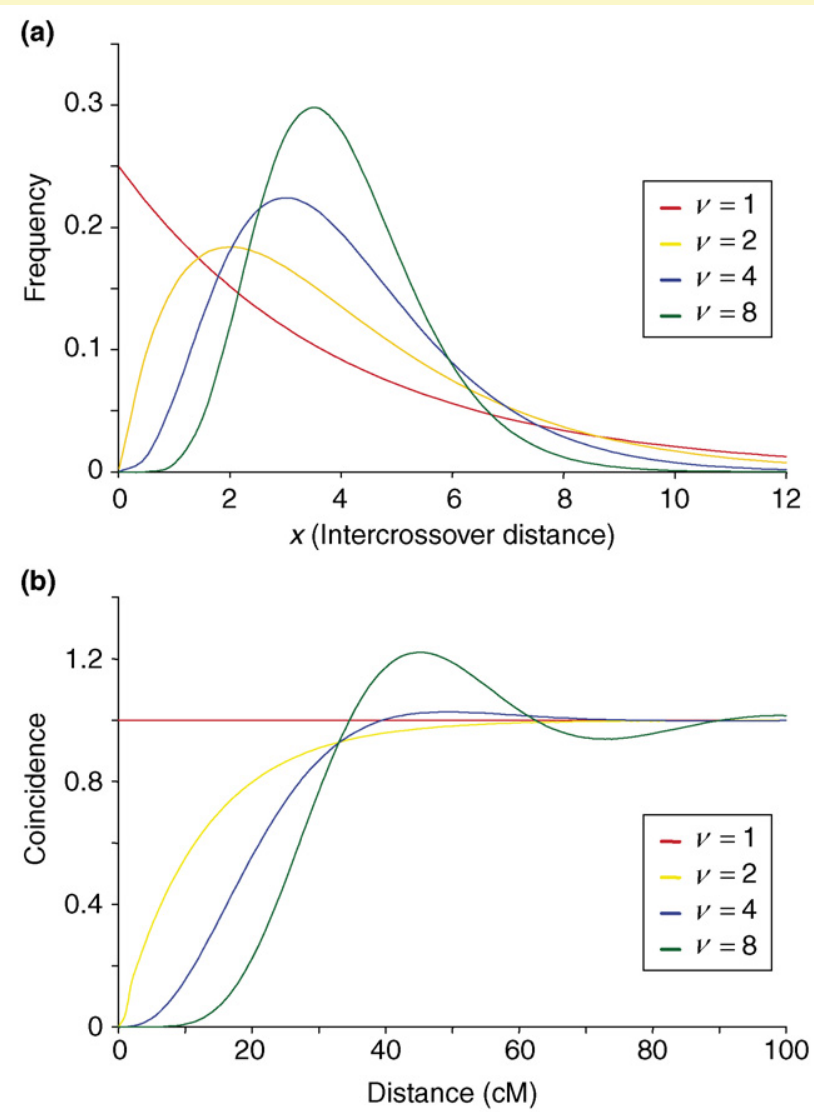

TRENDS in Genetics

Figure I. Using gamma distributions to determine the strength of interference. (a) Gamma distributions with varying shape $(\nu)$ and rate $(\lambda)$ parameters and a constant mean. (b) Coincidence functions deduced from gamma distributions with a varying shape $(\nu)$ parameter.

and Mms4 [43]. The actions of ZMM proteins indicate that interfering-crossover sites are selected from potential precursors at an early stage in the meiotic DSB-repair process [1,44]. In Arabidopsis, the depletion of AtMSH4 and AtMER3 (also known as MER3), two of the ZMM proteins, greatly reduces the number of crossovers that form, with atmsh 4 mutants having $85 \%$ fewer chiasmata than wildtype Arabidopsis [39] and atmer3 mutants having 75\% fewer $[38,40]$. The interference between the crossovers that are present is substantially reduced in atmer 3 mutants [40]. Therefore, the interfering pathway is responsible for the formation of most crossovers in Arabidopsis and involves AtMSH4 and AtMER3. Copenhaver et al. also estimated a proportion of $\sim 15 \%$ of noninterfering crossovers by fitting model data to genetic data [45].

Zip1, another ZMM protein in yeast, has a structural role in the synaptonemal complex $[42,46]$. It had been proposed that the polymerization of the synaptonemal complex could be the interference signal, but there is now strong evidence against the idea of such a mechanism 
$[1,47]$. Unlike the situation in yeast, in which the absence of Zip1 leads to a marked reduction in the number of crossovers, crossover formation is only slightly reduced (by 20\%) when the two Arabidopsis orthologs of yeast ZIP1 (ATZYP1A and ATZYP1B; also known as ZYP1A and $Z Y P 1 B)$ are inactivated using RNA interference [48]. Because no direct assay was carried out to study interference in this context, additional analysis is needed to confirm or to disprove the role of the AtZYP1 proteins in interference in Arabidopsis. In addition, multivalents and bivalents between nonhomologous chromosomes are observed when both ATZYP1 genes are inactivated, showing that the AtZYP1 proteins are required to restrain the formation of crossovers between homologous chromosomes [48]. These results indicate that, in Arabidopsis, AtZYP1A and AtZYP1B are not ZMM proteins as defined in yeast.

In yeast and Arabidopsis, other proteins also promote crossover formation (Figure 1). Meiotic crossover defects have been described in mlh1 [49] and mlh3 [50] deletion mutants in yeast and in atmlh3 [51] mutants in Arabidopsis but seem to be less severe than those in yeast msh4 or Arabidopsis atmsh4 mutants. The role of these factors in interference is either controversial (for yeast mlh1 and mlh3) [52] or unconfirmed (for Arabidopsis atmlh3), and their relationship to ZMM proteins is still unclear. In Arabidopsis, PARTING DANCERS (ATPTD; also known as AT1G12790.1), a novel gene that has no homolog in other kingdoms, might function in the interfering pathway: atptd mutants have $75 \%$ fewer chiasmata than wild-type Arabidopsis [41]. The list of genes implicated in crossover formation is growing, and it is unlikely that all the factors involved have been identified.

Three of the proteins involved in the formation of interfering crossovers have been immunolocalized in several species. In the mouse, MLH1 and MLH3 localize at crossover sites on meiotic chromosomes [53] and are commonly used to count crossovers, although it is unknown whether they mark all crossover sites. In tomato, there are $30 \%$ fewer MLH1 foci than LNs, and comparisons of their distributions indicate that interference among MLH1 foci is much stronger than among LNs. Because LNs correspond closely to chiasmata [31], this finding indicates that LNs mark all sites of crossovers (interfering and noninterfering), whereas MLH1 foci mark only a subset of strongly interfering crossovers. In addition, simulation shows that the distribution obtained when adding 30\% more crossovers, in random positions, to the observed distribution of MLH1 foci matches the observed distribution of LNs [54] (C. Heyting, unpublished). In Arabidopsis, AtMLH1 and AtMLH3 foci colocalize at pachytene [51]. These foci are slightly less numerous than the chiasmata count at metaphase I, which itself is probably a small underestimation of the number of crossovers. This suggests that these two proteins mark the sites of interfering crossovers and not the sites of noninterfering crossovers as in tomato. The situation is different for Msh4 and its orthologs in other species. In yeast, the number of Msh4 foci matches the number of crossovers [55]. By contrast, in the mouse and in Arabidopsis, numerous AtMSH4 foci appear at mid-leptotene, clearly marking more sites than there are crossovers [39,56]; the function of Msh4 and its orthologs at these sites still needs to be elucidated. Then, the number of these sites gradually decreases at zygotene and pachytene. In Arabidopsis, the colocalization of AtMLH3 and AtMSH4 is poor at pachytene [51], indicating that these proteins do not function at the same time in the formation of interfering crossovers.

\section{Noninterfering crossovers coexist with interfering crossovers}

Two factors in the noninterfering-crossover pathway have been identified in yeast: Mus81 and Mms4 [43] (Figure 1). These proteins are responsible for $\sim 30 \%$ of all crossovers. Arabidopsis contains two putative homologs of MUS81 [57], which might function in the noninterfering-crossover pathway.

The ratio of interfering crossovers to noninterfering crossovers differs among the species in which it has been studied. In yeast and tomato, $\sim 30 \%$ of crossovers seem to escape the interference mechanism; and in Arabidopsis, 15\%. At the two extremes are Caenorhabditis elegans, which has only interfering crossovers, and S. pombe (which lacks Msh4 and Msh5), which has only noninterfering crossovers [2]. Interestingly, in plants as diverse as potato, poplar, rice and Arabidopsis, homologs of the two sets of proteins (those involved in interfering crossovers and those involved in noninterfering crossovers) can be found, suggesting that the coexistence of the two pathways is a common feature in the plant kingdom. It is unclear why certain species have conserved both pathways and why the balance between the two pathways differs markedly. To add to this complexity, some crossovers still occur when both interfering-crossover (ZMM proteins) and noninterfering-crossover (Mus81 or Mms4) pathways are simultaneously disrupted in yeast [43,50], suggesting the existence of a third crossover-formation mechanism.

\section{Are there several layers of interference?}

That interference occurs between LNs, crossovers or Mlh1 foci at pachytene is no longer in doubt. Interestingly, a low level of interference has been detected between MSH4 foci (half that at MLH1 foci) at late zygotene in the mouse [58]. The distribution of ENs in tomato seems to show interference [58], in contrast to previous descriptions of a large range of plant species, for which the distribution of ENs was reported to be free of interference (except possibly over short distances) [22]. These new findings indicate that there are also two levels of interference in tomato. It is unknown whether these two putative levels of interference are mediated by two independent, still undefined, mechanisms and whether they are concomitant or are established successively as meiosis progresses. If ENs mark all DSB sites, then the first level of interference must be at the stage of DSB formation. At present, although it is clear that, in yeast, the density of DSBs varies enormously along chromosomes $[59,60]$, there are no data about putative interference between DSBs.

\section{Models of interference}

As it became clear that crossovers and noncrossovers both arise from meiotic DSBs and have a common early-recombination DNA structure, it was postulated that 


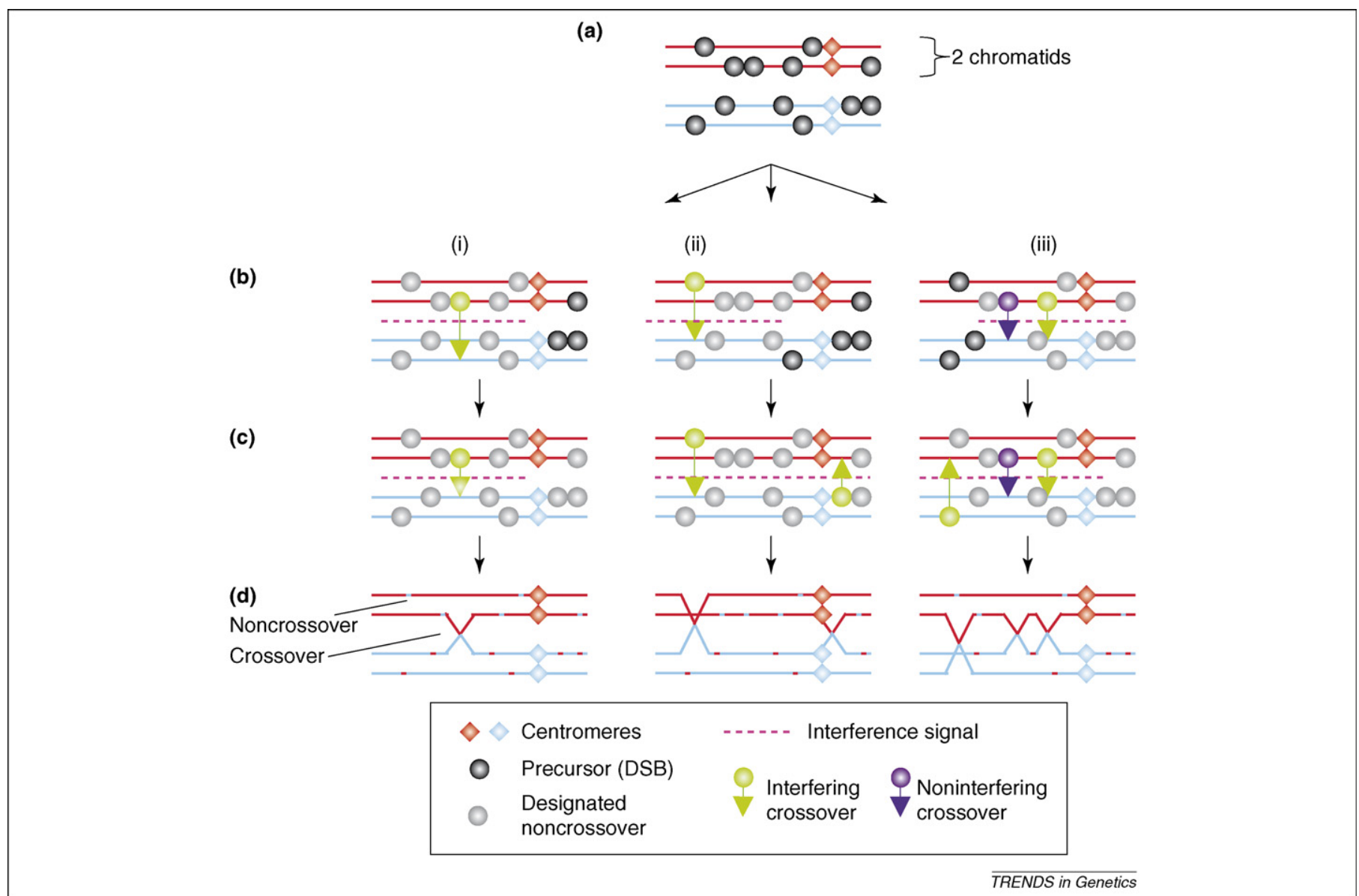

Figure 3. The process of crossover formation. The start point of crossover formation is a distribution of precursors (i.e. DSBs) among and along chromosomes (a). The density of these precursors varies along chromosomes, but how their distribution is controlled is largely unknown. These DSBs can be repaired as crossovers or noncrossovers. The tendency of individual precursors to become a crossover probably varies [28,29]. The mechanism that controls the decision of whether a precursor is repaired as a crossover or noncrossover is unknown. When one of the precursors, assumed to be stochastically chosen, is designated to become a crossover (b), this site inhibits the formation of a second crossover in its vicinity, implying that precursors subject to this effect become noncrossovers (c). The outcome is shown in (d). The way in which potential crossovers crosstalk to establish such interference at the chromosome level is not understood. The precursors located outside the effect of interference (i.e. the interference signal) might not become crossovers (i), or they can be designated as a second crossover and, in turn, inhibit the formation of other crossovers nearby (ii,iii). This controlled selection of a few sites to become crossovers from a large population of precursors (assuring that each bivalent has at least one crossover) explains the two main properties of crossover distribution: the obligatory crossover and interference. (For a discussion of the relationship between obligatory chiasmata and interference, see Ref. [80].) A proportion of precursors can be designated to become noninterfering crossovers, independent of the presence of another crossover nearby (iii). The way in which some precursors enter this pathway, and why they are not subject to interference, is still puzzling.

interference is imposed when they diverge [61] (Figure 3). This led to the proposal of a series of models to explain the control of the differentiation between crossovers and noncrossovers and the coordination at the chromosome level of this control.

In the counting model, the number of noncrossover events between crossovers $(m)$ is fixed. Therefore, $m$ directly reflects the strength of interference (Box 2). The fit of the counting model to genetic data sets is satisfactory in most cases [62], so this became one of the main models in the field. However, the basis for fixing the value of $m$ is challenged by numerous data indicating that $m$ varies between sexes and individuals in the same species, between chromosomes in the same nucleus and even along chromosomes [63,64]. To account for the variations in strength of interference, two modified versions of the counting model were proposed: one in which $m$ is not constant [65], and a second in which the existence of noninterfering crossovers is taken into account [33,3537]. In this second model, the apparent variation in interference strength is due to the variable proportion of noninterfering crossovers to interfering crossovers $(p)$ from chromosome to chromosome and along chromosomes, rather than to variation in the mechanism of interference itself $(m)$. Indeed, this assumption improves the fit of the model to the genetic data from yeast, humans and Arabidopsis [33,35-37]. However, the best fitting values for $m$ and $p$ in Arabidopsis [33,37] predict that the crossovers that occur in ZMM protein mutants will not be distributed equally among chromosomes. This prediction is not substantiated in atmsh4 [39] and atmer3 mutants (J. Vignard and R. Mercier, unpublished), indicating that a variation in the ratio of noninterfering crossovers to interfering crossovers is insufficient to account for the variation in interference between chromosomes. Another set of data obtained recently in yeast also presents serious challenges to the counting model. Indeed, the decrease in the number of meiotic DSBs was shown not to reduce the number of crossovers proportionally (a phenomenon known as crossover homeostasis) and not to modify the strength of interference [66]. This contradicts the prediction of the counting model, which finds that to keep the number of 
noncrossovers constant between two crossovers $(m)$, the number of crossovers needs to decrease.

Another model postulates that early recombination intermediates sometimes become crossovers and then nucleate an inhibitory polymerization; this interference signal then propagates in its own vicinity and forces other early events to resolve as noncrossovers [67]. The more obvious candidate for the propagation of this signal was the polymerization of the synaptonemal complex, but there is now strong evidence against the idea of such a mechanism [1,47]. No other potential mediator of this signal has been identified.

More recently, in another model, known as the mechanical stress model, the designation of crossovers among DSBs results from the imposition of a physical stress on the chromatin fiber [68]. The occurrence of a crossover results in local relief of the stress, which spreads out in both directions from the affected site with decreasing strength. Therefore, the probability of a second crossover occurring adjacent to the first is zero and progressively increases with distance, up to the initial probability. A simulation according to this model successfully fitted genetic data from two species. However, there has been no in vivo demonstration supporting this model.

\section{Conclusions}

As more genetic and cytological data become available, in particular data from mutants of various species, the emerging picture of crossover formation is becoming more detailed and is turning out to be more complex than was expected a few years ago. The final distribution of crossovers results from the integration of several levels of control (Figure 3), which can be summarized as follows: (i) the density of DSBs varies along chromosomes in yeast, and probably in other species, and DSBs might interfere; (ii) the propensity of a DSB to become a crossover or a noncrossover (or an SCE) probably varies; (iii) interference might operate at several levels, and its strength varies along a chromosome and among chromosomes; (iv) some crossovers, the proportion of which differs among species, are insensitive to interference; and (v) there might be more than two pathways controlling crossover formation.

At present, each of these different levels of control of crossover formation is poorly understood, and there is still no unified theory of crossover differentiation that integrates genetic and cytological data and proposes a satisfactory underlying molecular mechanism. However, there is much research activity in the field, and communication between groups working on a large range of model species in different kingdoms has recently become intense. Plants have their say in this dialog thanks to the combination of available genetic data and superb cytological tools for studying several species, in addition to the molecular mechanism of crossover formation having been deciphered, particularly in Arabidopsis. This multi-organism approach should soon result in new insights into the mechanisms involved in each level of control of the final crossover distribution.

\section{Acknowledgements}

We thank Françoise Budar, Mathilde Grelon, Eric Jenczewski and Olivier Loudet for critical reading of the manuscript. Many thanks also to Christa Heyting for sharing data before publication.

\section{References}

1 Bishop, D.K. and Zickler, D. (2004) Early decision; meiotic crossover interference prior to stable strand exchange and synapsis. Cell 117, 9 15

2 Hollingsworth, N.M. and Brill, S.J. (2004) The Mus81 solution to resolution: generating meiotic crossovers without Holliday junctions. Genes Dev. 18, 117-125

3 Jones, G.H. (1984) The control of chiasma distribution. Symp. Soc. Exp. Biol. 38, 293-320

4 Lynn, A. et al. (2004) Variation in human meiotic recombination. Annu. Rev. Genomics Hum. Genet. 5, 317-349

5 Bateson, W. (1909) Mendel's Principles of Heredity, Cambridge University Press

6 Keeney, S. (2001) Mechanism and control of meiotic recombination initiation. Curr. Top. Dev. Biol. 52, 1-53

7 Schwacha, A. and Kleckner, N. (1997) Interhomolog bias during meiotic recombination: meiotic functions promote a highly differentiated interhomolog-only pathway. Cell 90, 1123-1135

8 Grelon, M. et al. (2001) AtSPO11-1 is necessary for efficient meiotic recombination in plants. $E M B O \mathrm{~J} .20,589-600$

9 Stacey, N.J. et al. (2006) Arabidopsis SPO11-2 functions with SPO11-1 in meiotic recombination. Plant J. 48, 206-216

10 Bleuyard, J.Y. et al. (2004) Meiotic defects in the Arabidopsis rad50 mutant point to conservation of the MRX complex function in early stages of meiotic recombination. Chromosoma 113, 197-203

11 Puizina, J. et al. (2004) Mre11 deficiency in Arabidopsis is associated with chromosomal instability in somatic cells and Spo11dependent genome fragmentation during meiosis. Plant Cell 16, 19681978

12 Jolivet, S. et al. (2006) Non conservation of the meiotic function of the Ski8/Rec103 homolog in Arabidopsis. Genes Cells 11, 615-622

13 Stack, S.M. et al. (1989) Chiasmata and recombination nodules in Lilium longiflorum. Genome 32, 486-498

14 Anderson, L.K. and Stack, S.M. (2005) Recombination nodules in plants. Cytogenet. Genome Res. 109, 198-204

15 Zickler, D. and Kleckner, N. (1999) Meiotic chromosomes: integrating structure and function. Annu. Rev. Genet. 33, 603-754

16 Anderson, L.K. et al. (1997) RecA-like proteins are components of early meiotic nodules in lily. Proc. Natl. Acad. Sci. U. S. A. 94, 6868-6873

$17 \mathrm{Li}$, W. et al. (2004) The Arabidopsis AtRAD51 gene is dispensable for vegetative development but required for meiosis. Proc. Natl. Acad. Sci. U. S. A. 101, 10596-10601

18 Couteau, F. et al. (1999) Random chromosome segregation without meiotic arrest in both male and female meiocytes of a dmc1 mutant of Arabidopsis. Plant Cell 11, 1623-1634

19 Siaud, N. et al. (2004) Brca2 is involved in meiosis in Arabidopsis thaliana as suggested by its interaction with Dmc1. EMBO J. 23, 13921401

20 Mezard, C. (2006) Meiotic recombination hot spots in plants. Biochem. Soc. Trans. 34, 531-534

21 Choo, K.H. (1998) Why is the centromere so cold? Genome Res. 8, 81-82

22 Anderson, L.K. et al. (2001) The distribution of early recombination nodules on zygotene bivalents from plants. Genetics 159, 12591269

23 Moens, P.B. et al. (2002) The time course and chromosomal localization of recombination-related proteins at meiosis in the mouse are compatible with models that can resolve the early DNA-DNA interactions without reciprocal recombination. J. Cell Sci. 115, 1611-1622

24 Chelysheva, L. et al. (2005) AtREC8 and AtSCC3 are essential to the monopolar orientation of the kinetochores during meiosis. J. Cell Sci. $118,4621-4632$

25 Plagnol, V. et al. (2005) Relative influences of crossing-over and gene conversion on the pattern of linkage disequilibrium in Arabidopsis thaliana. Genetics 172, 2441-2448

26 Stack, S.M. and Anderson, L.K. (2002) Crossing over as assessed by late recombination nodules is related to the pattern of synapsis and the distribution of early recombination nodules in maize. Chromosome Res. $10,329-345$

27 Fogel, S. et al. (1981) Mechanisms of meiotic gene conversion, or 'wanderings on a foreign strand'. In The Molecular Biology of the Yeast Saccharomyces: Life Cycle and Inheritance (Strathern, J. et al., eds), pp. 289-339, Cold Spring Harbor Laboratory Press 
28 Jeffreys, A.J. and Neumann, R. (2005) Factors influencing recombination frequency and distribution in a human meiotic crossover hotspot. Hum. Mol. Genet. 37, 601-606

29 Kauppi, L. et al. (2004) Where the crossovers are: recombination distributions in mammals. Nat. Rev. Genet. 5, 413-424

30 Muller, H.J. (1916) The mechanisms of crossing-over. Am. Nat. 50, 193-221

31 Sherman, J.D. and Stack, S.M. (1995) Two-dimensional spreads of synaptonemal complexes from solanaceous plants. VI. High-resolution recombination nodule map for tomato (Lycopersicon esculentum). Genetics 141, 683-708

32 Anderson, L.K. et al. (2003) High-resolution crossover maps for each bivalent of Zea mays using recombination nodules. Genetics 165, 849-865

33 Lam, S.Y. et al. (2005) Crossover interference on nucleolus organizing region-bearing chromosomes in Arabidopsis. Genetics 170, 807-812

34 Malkova, A. et al. (2004) Gene conversion and crossing over along the 405-kb left arm of Saccharomyces cerevisiae chromosome VII. Genetics $168,49-63$

35 Stahl, F.W. et al. (2004) Does crossover interference count in Saccharomyces cerevisiae? Genetics 168, 35-48

36 Housworth, E.A. and Stahl, F.W. (2003) Crossover interference in humans. Am. J. Hum. Genet. 73, 188-197

37 Copenhaver, G.P. et al. (2002) Crossover interference in Arabidopsis. Genetics 160, 1631-1639

38 Chen, C.et al. (2005) The Arabidopsis ROCK-N-ROLLERS gene encodes a homolog of the yeast ATP-dependent DNA helicase MER3 and is required for normal meiotic crossover formation. Plant J. 43, 321-334

39 Higgins, J.D. et al. (2004) The Arabidopsis MutS homolog AtMSH4 functions at an early step in recombination: evidence for two classes of recombination in Arabidopsis. Genes Dev. 18, 2557-2570

40 Mercier, R. et al. (2005) Two meiotic crossover classes cohabit in Arabidopsis: one is dependent on MER3, whereas the other one is not. Curr. Biol. 15, 692-701

41 Wijeratne, A.J. et al. (2006) The Arabidopsis thaliana PARTING DANCERS gene encoding a novel protein is required for normal meiotic homologous recombination. Mol. Biol. Cell 17, 1331-1343

42 Borner, G.V. et al. (2004) Crossover/noncrossover differentiation, synaptonemal complex formation, and regulatory surveillance at the leptotene/zygotene transition of meiosis. Cell 117, 29-45

43 De Los Santos, T. et al. (2003) The Mus81/Mms4 endonuclease acts independently of double-Holliday junction resolution to promote a distinct subset of crossovers during meiosis in budding yeast. Genetics 164, 81-94

44 Fung, J.C. et al. (2004) Imposition of crossover interference through the nonrandom distribution of synapsis initiation complexes. Cell 116, 795-802

45 Copenhaver, G.P. et al. (1998) Assaying genome-wide recombination and centromere functions with Arabidopsis tetrads. Proc. Natl. Acad. Sci. U. S. A. 95, 247-252

46 Sym, M. et al. (1993) ZIP1 is a synaptonemal complex protein required for meiotic chromosome synapsis. Cell 72, 365-378

47 de Boer, E. and Heyting, C. (2006) The diverse roles of transverse filaments of synaptonemal complexes in meiosis. Chromosoma 115, $220-234$

48 Higgins, J.D. et al. (2005) The Arabidopsis synaptonemal complex protein ZYP1 is required for chromosome synapsis and normal fidelity of crossing over. Genes Dev. 19, 2488-2500

49 Wang, T.F. et al. (1999) Functional specificity of MutL homologs in yeast: evidence for three Mlh1-based heterocomplexes with distinct roles during meiosis in recombination and mismatch correction. Proc. Natl. Acad. Sci. U. S. A. 96, 13914-13919

50 Argueso, J.L. et al. (2004) Competing crossover pathways act during meiosis in Saccharomyces cerevisiae. Genetics 168, 1805-1816

51 Jackson, N. et al. (2006) Reduced meiotic crossovers and delayed prophase I progression in AtMLH3-deficient Arabidopsis. EMBO J. $25,1315-1323$

52 Hoffmann, E.R. and Borts, R.H. (2004) Meiotic recombination intermediates and mismatch repair proteins. Cytogenet. Genome Res. 107, 232-248
53 Marcon, E. and Moens, P. (2003) MLH1p and MLH3p localize to precociously induced chiasmata of okadaic-acid-treated mouse spermatocytes. Genetics 165, 2283-2287

54 Lhuissier, F.G.P. et al. (2005) An immunocytological recombination map of tomato. In The European Meiosis Meeting, p. 104, European Molecular Biology Organization

55 Novak, J.E. et al. (2001) The budding yeast Msh4 protein functions in chromosome synapsis and the regulation of crossover distribution. Genetics 158, 1013-1025

$56 \mathrm{Kneitz,} \mathrm{B.} \mathrm{et} \mathrm{al.} \mathrm{(2000)} \mathrm{MutS} \mathrm{homolog} 4$ localization to meiotic chromosomes is required for chromosome pairing during meiosis in male and female mice. Genes Dev. 14, 1085-1097

57 Hartung, F. et al. (2006) The role of AtMUS81 in DNA repair and its genetic interaction with the helicase AtRecQ4A. Nucleic Acids Res. 34, $4438-4448$

58 de Boer, E. et al. (2006) Two levels of interference in mouse meiotic recombination. Proc. Natl. Acad. Sci. U. S. A. 103, 96079612

59 Gerton, J.L. et al. (2000) Global mapping of meiotic recombination hotspots and coldspots in the yeast Saccharomyces cerevisiae. Proc. Natl. Acad. Sci. U. S. A. 97, 11383-11390

60 Baudat, F. and Nicolas, A. (1997) Clustering of meiotic double-strand breaks on yeast chromosome III. Proc. Natl. Acad. Sci. U. S. A. 94, 5213-5218

61 Mortimer, R.K. and Fogel, S. (1974) Genetical interference and gene conversion. In Mechanism in Recombination (Grell, R.F., ed.), pp. 263275, Plenum Press

62 Foss, E. et al. (1993) Chiasma interference as a function of genetic distance. Genetics 133, 681-691

63 Esch, E. (2005) Estimation of gametic frequencies from $\mathrm{F}_{2}$ populations using the EM algorithm and its application in the analysis of crossover interference in rice. Theor. Appl. Genet. 111, 100-109

64 Lin, S. et al. (2001) Genetic crossover interference in the human genome. Ann. Hum. Genet. 65, 79-93

65 Lange, K. et al. (1997) The Poisson-skip model of crossing-over. Ann. Appl. Probab. 7, 299-313

66 Martini, E. et al. (2006) Crossover homeostasis in yeast meiosis. Cell $126,285-295$

67 King, J.S. and Mortimer, R.K. (1990) A polymerization model of chiasma interference and corresponding computer simulation. Genetics 126, 1127-1138

68 Kleckner, N. et al. (2004) A mechanical basis for chromosome function. Proc. Natl. Acad. Sci. U. S. A. 101, 12592-12597

69 Mendel, G. (1866) Versuche über pflanzenhybriden. Verh. Naturf. Ver. Brünn. 4, 3-44

70 Whitehouse, H.L.K. (1973) Towards an Understanding of the Mechanisms of Heredity, (3rd edn), Edward Arnold

71 Bateson, W. et al. (1905) Experimental studies in the physiology of heredity. Rep. Evol. Comm. R. Soc. 2, 1-154

72 Creighton, H.B. and McClintock, B. (1931) A correlation of cytological and genetical crossing-over in Zea mays. Proc. Natl. Acad. Sci. U. S. A. $17,492-497$

73 Haldane, J.B.S. (1931) The cytological basis of genetical interference. Cytologia (Tokyo) 3, 54-65

74 McClintock, B. (1956) Controlling elements and the gene. Cold Spring Harbor Symp. Quant. Biol. 21, 197-216

75 Vaucheret, H. (2006) Post-transcriptional small RNA pathways in plants: mechanisms and regulations. Genes Dev. 20, 759-771

76 Colombo, P.C. and Jones, G.H. (1997) Chiasma interference is blind to centromeres. Heredity 79, 214-227

77 McPeek, M.S. and Speed, T.P. (1995) Modeling interference in genetic recombination. Genetics 139, 1031-1044

78 Hamant, O. et al. (2006) Genetics of meiotic prophase I in plants. Annu. Rev. Plant Biol. 57, 267-302

79 Drouaud, J. et al. (2006) Variation in crossing-over rates across chromosome 4 of Arabidopsis thaliana reveals the presence of meiotic recombination 'hot spots'. Genome Res. 16, 106-114

80 Jones, G.H. and Franklin, F.C. (2006) Meiotic crossing-over: obligation and interference. Cell 126, 246-248 